Supporting Information

\title{
Pore-Confined Silver Nanoparticles in a Porphyrinic Metal-Organic Framework for Electrochemical Nitrite Detection
}

Yi-Ching Wang, ‘ Yu-Chuan Chen, '† Wen-Shuo Chuang, ${ }^{\dagger}$ Jun-Hong Li, ${ }^{\dagger}$ Yi-Sen Wang, ${ }^{\dagger}$ Cheng-Hsun Chuang, ${ }^{\dagger}$

Chih-Yen Chen, ${ }^{*}$ and Chung-Wei Kung*, ${ }^{*}$

†Department of Chemical Engineering, National Cheng Kung University, 1 University Road, Tainan City, Taiwan.

^Department of Material Science and Optoelectronic Engineering, National Sun Yat-Sen University, Kaohsiung City, Taiwan.

\footnotetext{
* Corresponding authors: cwkung@mail.ncku.edu.tw ～(C.-W. K.)
}
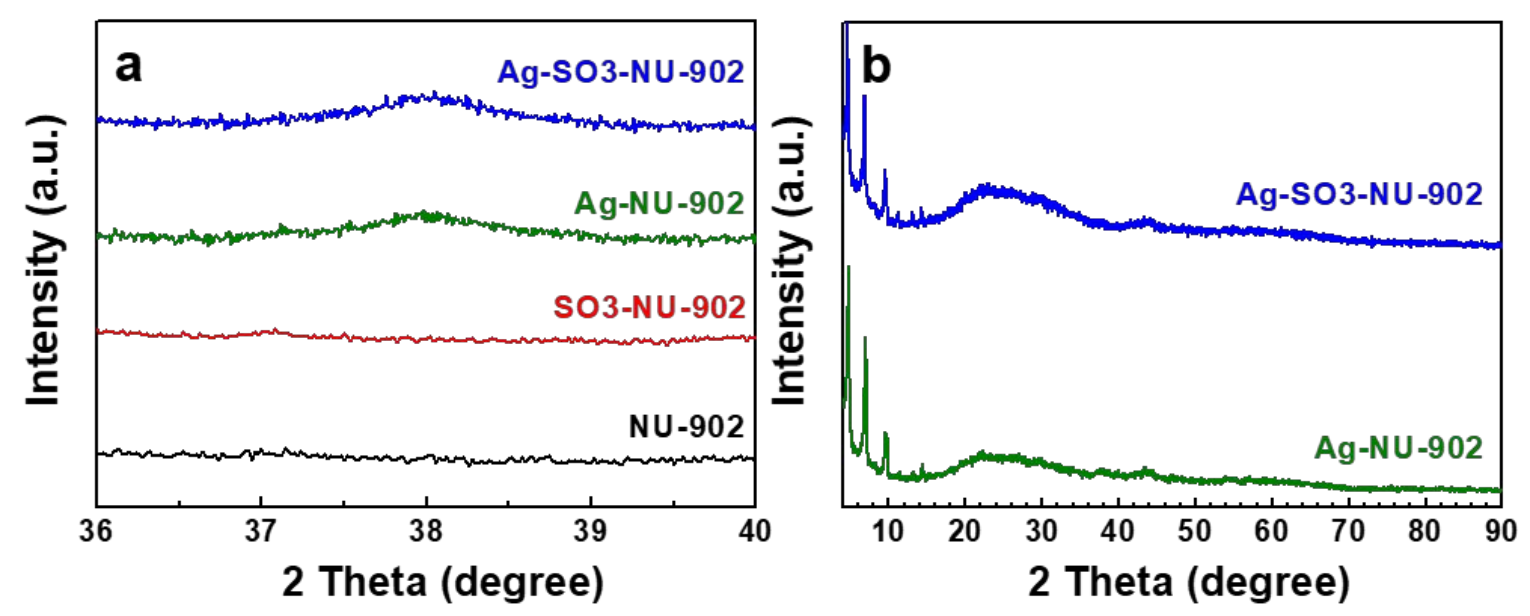

Figure S1. (a) XRD patterns of NU-902, SO3-NU-902, Ag-NU-902, and Ag-SO3-NU-902, collected from $36^{\circ}$ to $40^{\circ}$ at a slow scan speed of 0.1 degree/min. (b) Full-range XRD patterns of Ag-NU-902 and Ag-SO3-NU-902 measured at 1.0 degree/min. 

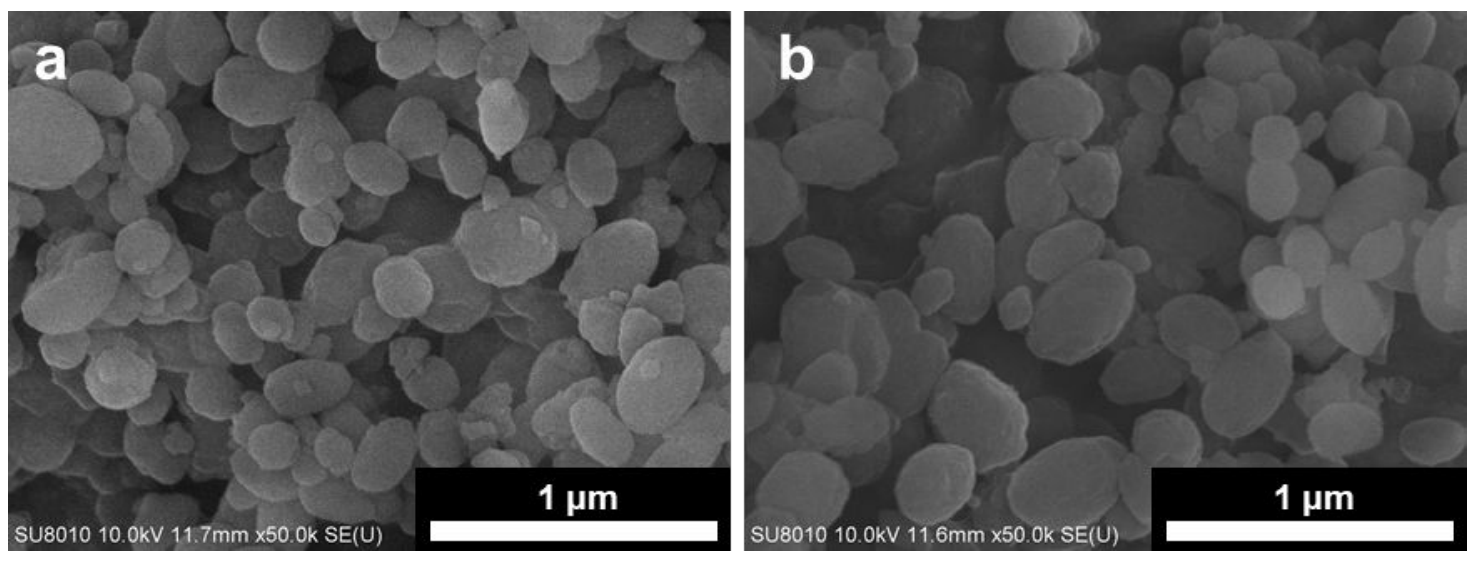

Figure S2. SEM images of (a) NU-902, and (b) SO3-NU-902.
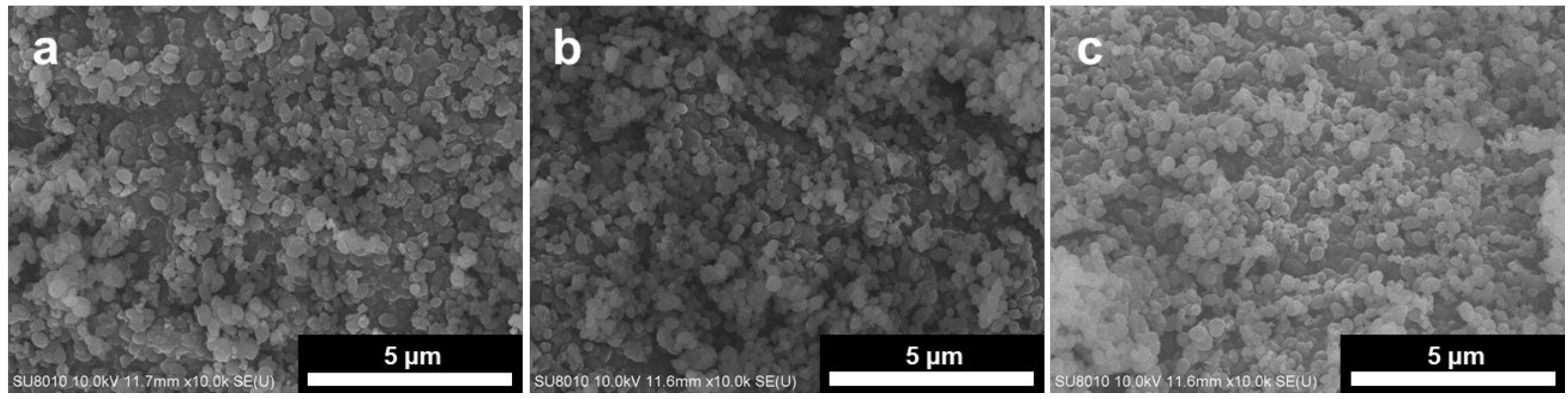

Figure S3. Low-magnification SEM images of (a) NU-902, (b) SO3-NU-902, and (c) AgSO3-NU-902. 

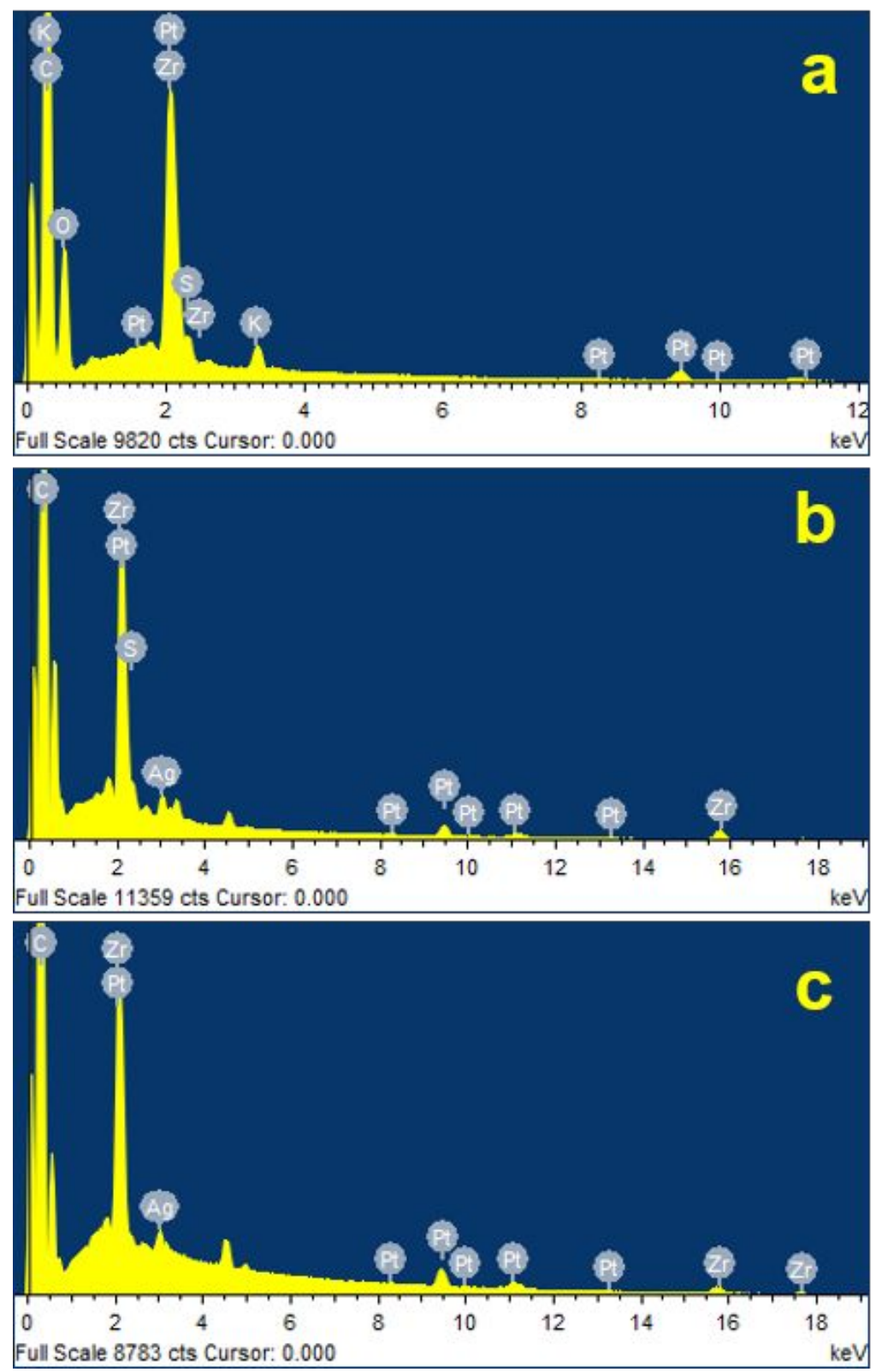

Figure S4. Representative EDS spectra of (a) SO3-NU-902, (b) Ag-SO3-NU-902, and (c) Ag-NU-902. 
To examine the equilibrium between $\mathrm{K}^{+}$and $\mathrm{Ag}^{+}$during the ion-exchange process, 18 crown- 6 with the amount of around six equivalents to the amount of $\mathrm{K}^{+}$presented in the added SO3-NU-902 sample was dissolved in the solution for the ion-exchange process. After the same ion-exchange process for overnight, the obtained solid was also washed with acetonitrile for three times followed by being washed with acetone for three times over the course of $24 \mathrm{~h}$, as mentioned in the experimental procedure of the ion-exchange process in the main text. After drying, the obtained solid was subjected to EDS measurements. According to the resulting spectra (see Figure S5(a)), the average loadings of 0.11 potassium atoms and 1.03 silver atoms on per hexa-zirconium node were obtained, respectively. The finding here suggests that with the help of 18-crown- 6 to remove the potassium ions during the ion-exchange process, the equilibrium between $\mathrm{K}^{+}$and $\mathrm{Ag}^{+}$can be shifted to achieve a higher silver loading in the resulting material. The sample was then digested for ${ }^{1} \mathrm{H}$ NMR analysis. However, as shown in Figure S5(b), a significant singlet peak located at around 3.4 ppm can be observed, which is originated from the twenty-four protons in 18-crown-6. This observation indicates that even after multiple washing steps with the total immersing period of $24 \mathrm{~h}$ in between, the 18-crown- 6 can not be removed from the MOF sample. As the 18crown-6 is presented in the sample as an extra impurity and can not be removed completely, we decided not to focus on this sample synthesized from the ion-exchange process with 18crown-6 in the following studies. 


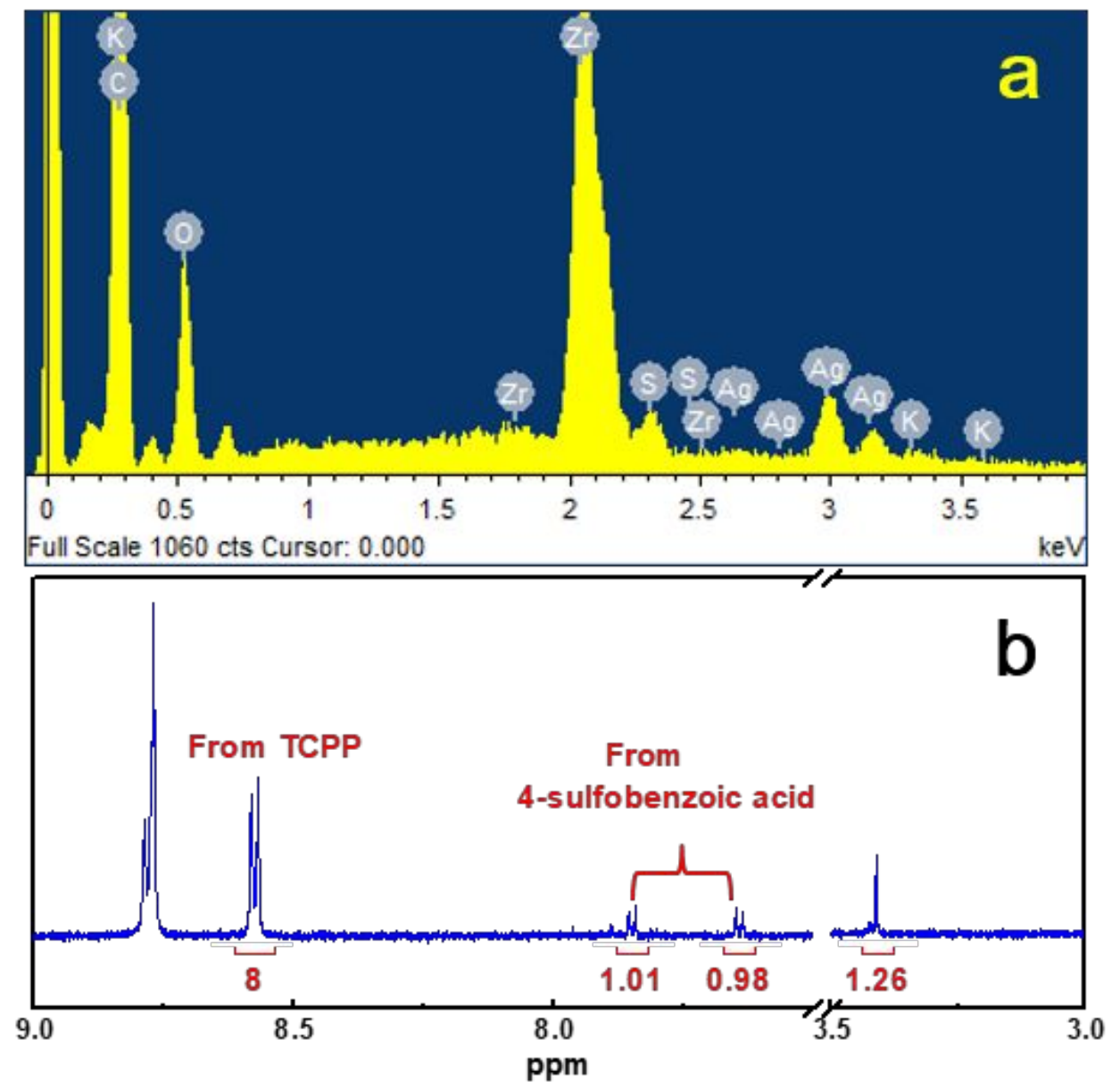

Figure S5. (a) EDS spectrum and (b) ${ }^{1} \mathrm{H}$ NMR spectrum of the silver incorporated SO3-NU902 sample synthesized from the ion-exchange process with the addition of 18-crown-6. 
To precisely quantify the amounts of sulfonate-based ligand installed within SO3-NU902 and Ag-SO3-NU-902, digestion of MOF sample was performed in concentrated $\mathrm{D}_{2} \mathrm{SO}_{4}$ for NMR measurements. The obtained ${ }^{1} \mathrm{H}$ NMR spectra are shown in Figure S6. The doublet peaks located at around $8.5 \mathrm{ppm}$ correspond to eight protons from the TCPP linker, ${ }^{1}$ and each of the doublet peaks located at around $7.7 \mathrm{ppm}$ correspond to two protons from the 4sulfobenzoic acid, respectively. Since the ratio between hexa-zirconium node and TCPP linker is 1:2 in the structure of NU-902, the average loadings of the sulfonate-based ligand in SO3-NU-902 and Ag-SO3-NU-902 are 1.34 and 0.85 ligand on per hexa-zirconium node, respectively.

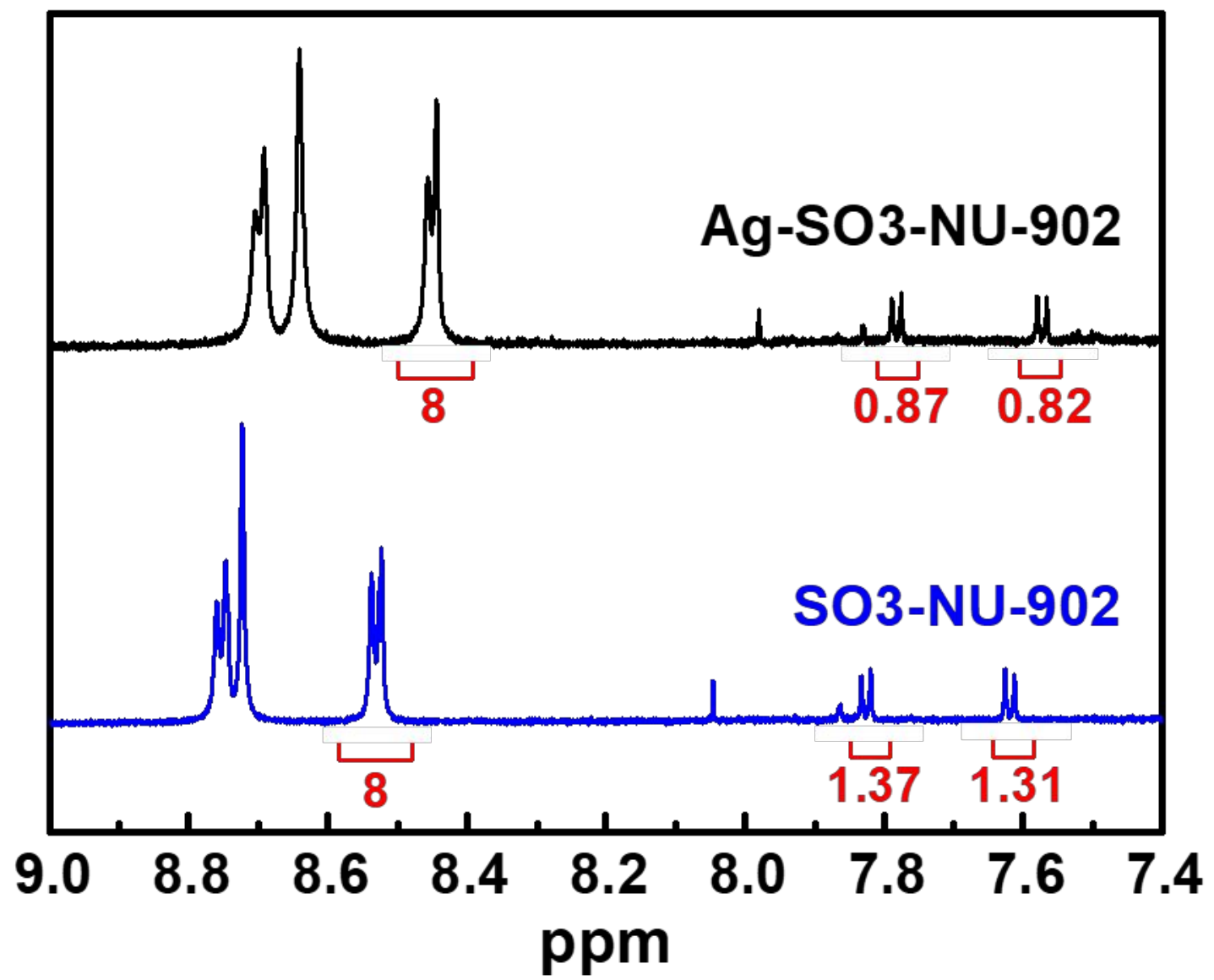

Figure S6. ${ }^{1} \mathrm{H}$ NMR spectra of digested SO3-NU-902 and Ag-SO3-NU-902. 
The NU-902, Ag-NU-902, and Ag-SO3-NU-902 were digested in 0.1 M NaOH aqueous solutions, and the obtained solutions were subjected to UV-vis measurements. As revealed in Figure S7, all the three digested samples show the same UV-vis characteristic with a Soret band located at the same wavelength and four Q bands; this characteristic agrees well with the typical characteristic of free-base TCPP. ${ }^{2-3}$ This observation suggests that the metalation of silver onto the TCPP linkers did not occur during the preparation of both Ag-NU-902 and Ag-SO3-NU-902.

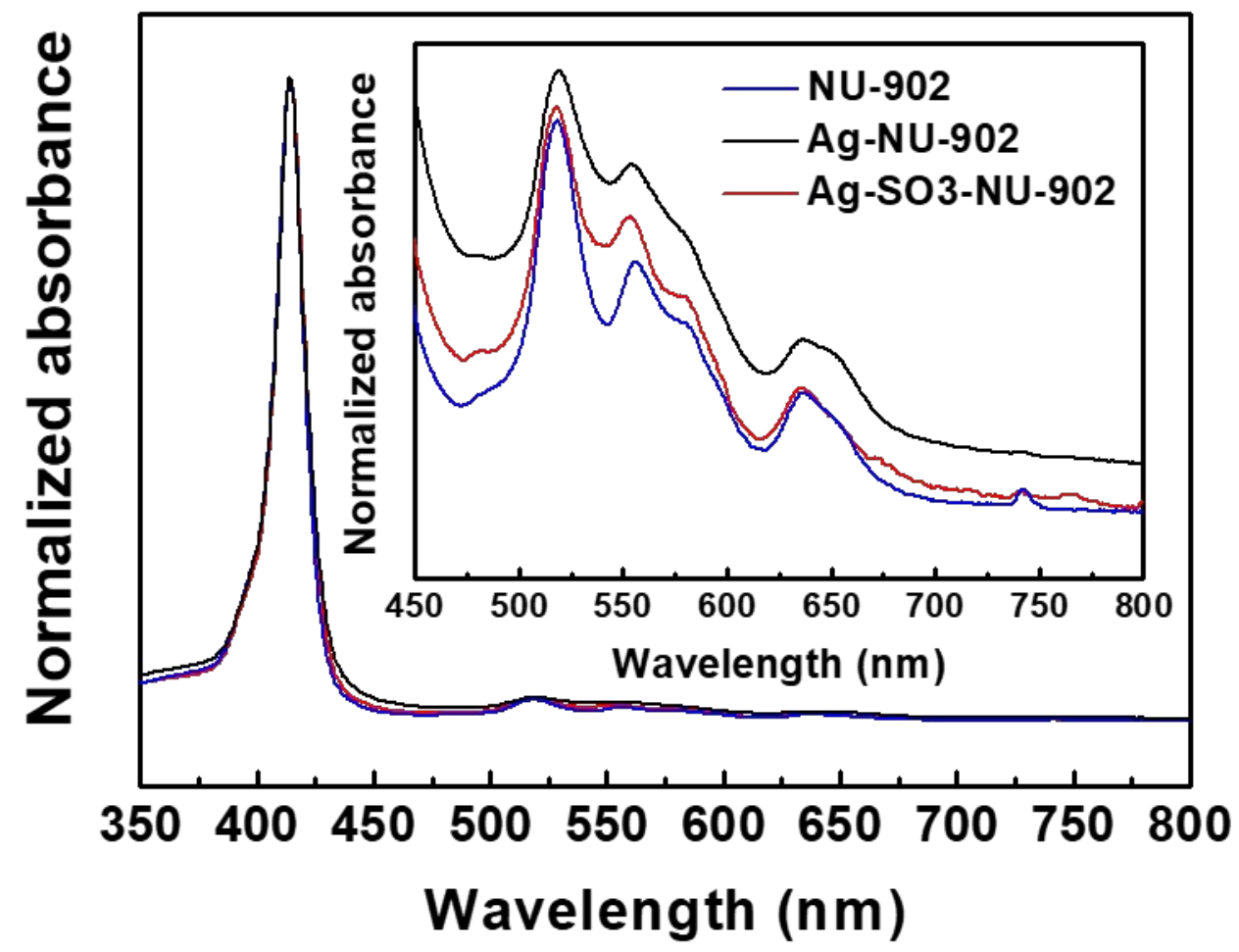

Figure S7. UV-vis spectra of the digested NU-902, Ag-NU-902, and Ag-SO3-NU-902. 


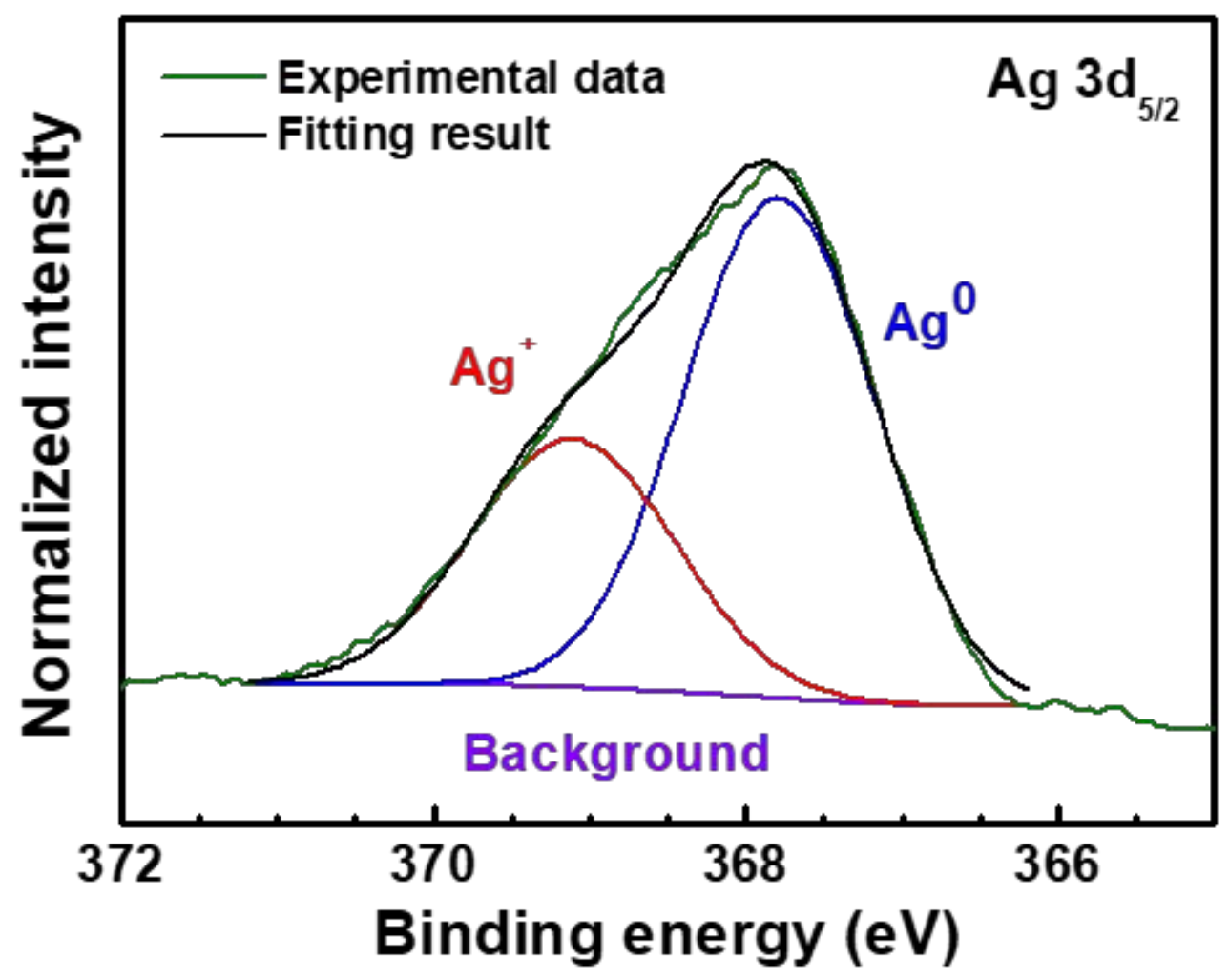

Figure S8. XPS spectrum of Ag-NU-902 in the region of $\mathrm{Ag} 3 \mathrm{~d}_{5 / 2}$.
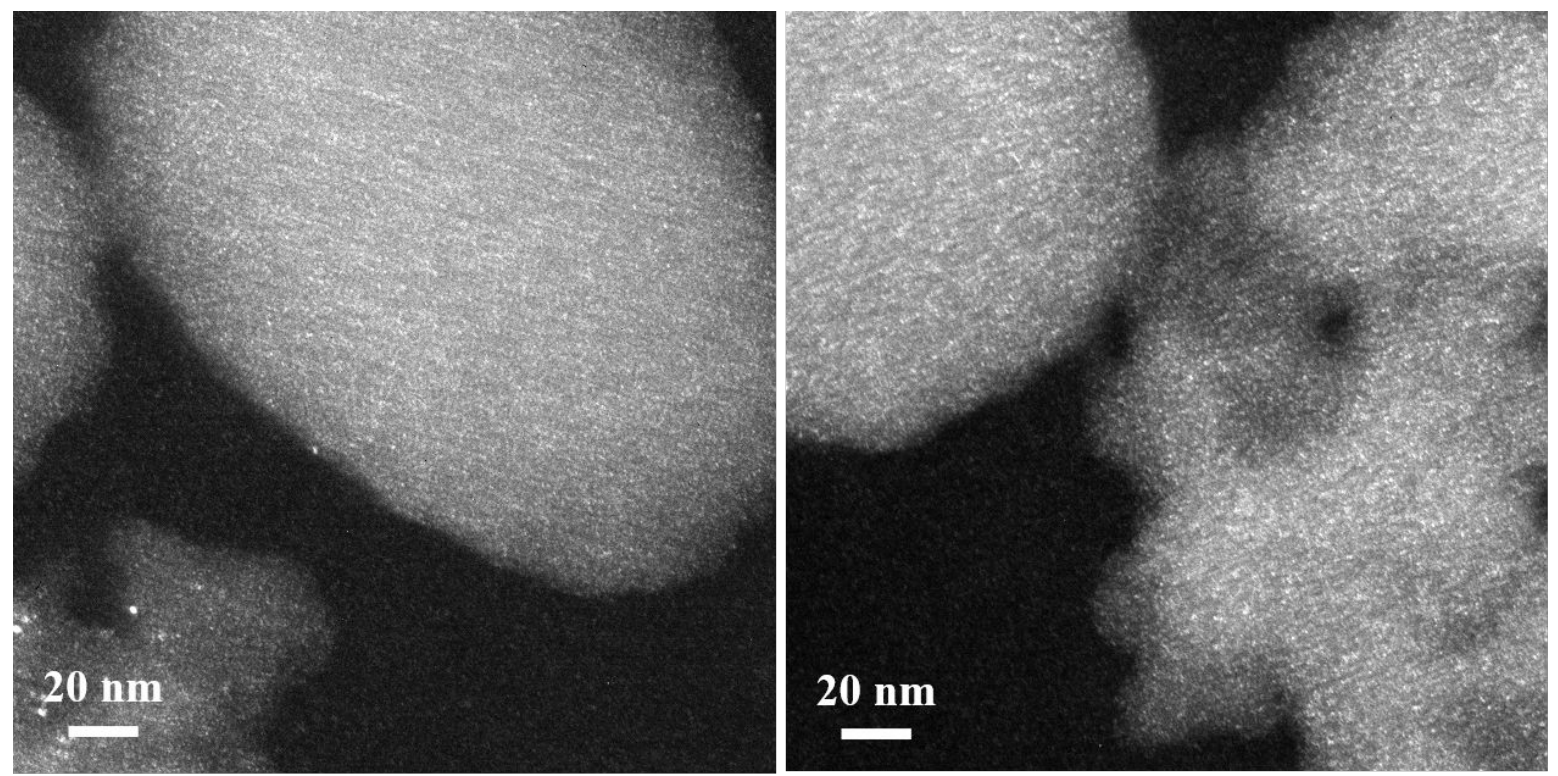

Figure S9. Dark-field TEM images of Ag-SO3-NU-902. 

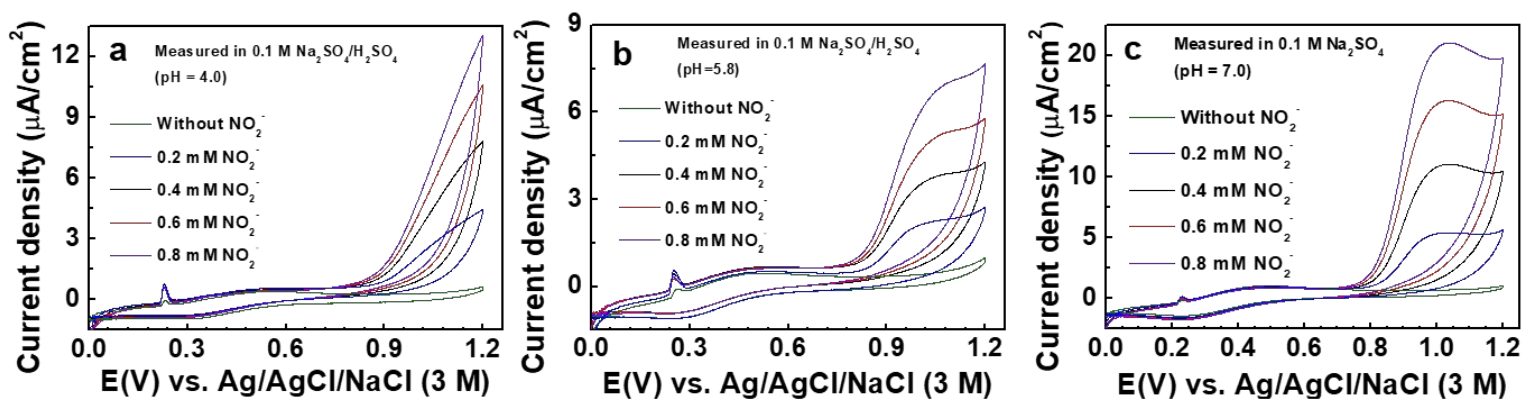

Figure S10. CV curves of Ag-SO3-NU-902 thin films measured in $0.1 \mathrm{M} \mathrm{Na}_{2} \mathrm{SO}_{4}$-based aqueous solutions with the $\mathrm{pH}$ values of (a) 4.0, (b) 5.8, and (c) 7.0, containing various concentrations of nitrite at a scan rate of $10 \mathrm{mV} / \mathrm{s}$.
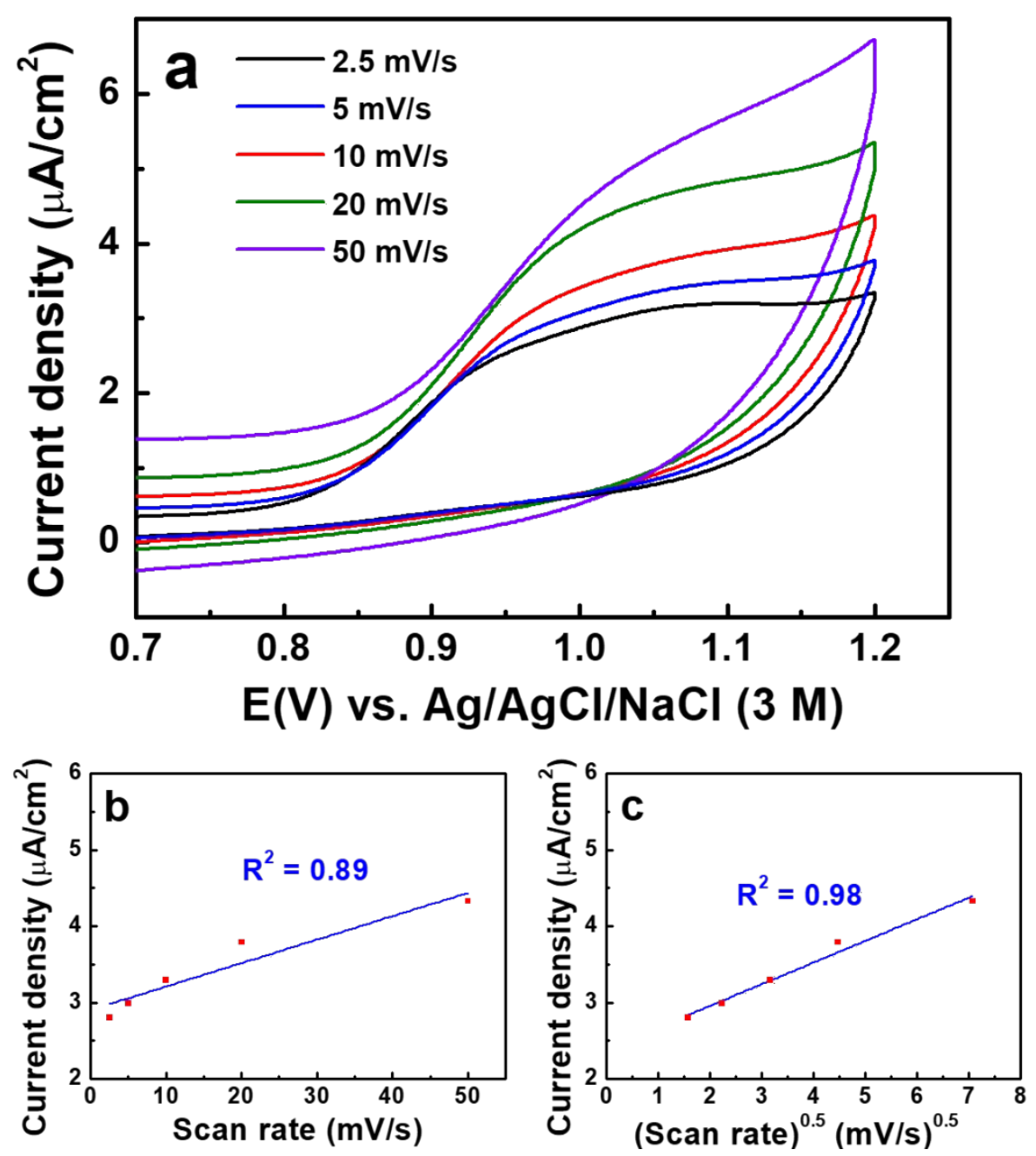
Figure S11. (a) CV curves of the Ag-SO3-NU-902 thin film measured in the $0.1 \mathrm{M} \mathrm{Na}_{2} \mathrm{SO}_{4}$ aqueous solution containing $0.2 \mathrm{mM}$ of nitrite at various scan rates. (b) Plot of anodic peak current density vs. scan rate extracted from the CV curves in (a). (c) Plot of anodic peak current density vs. (scan rate) $)^{0.5}$ extracted from the CV curves in (a).

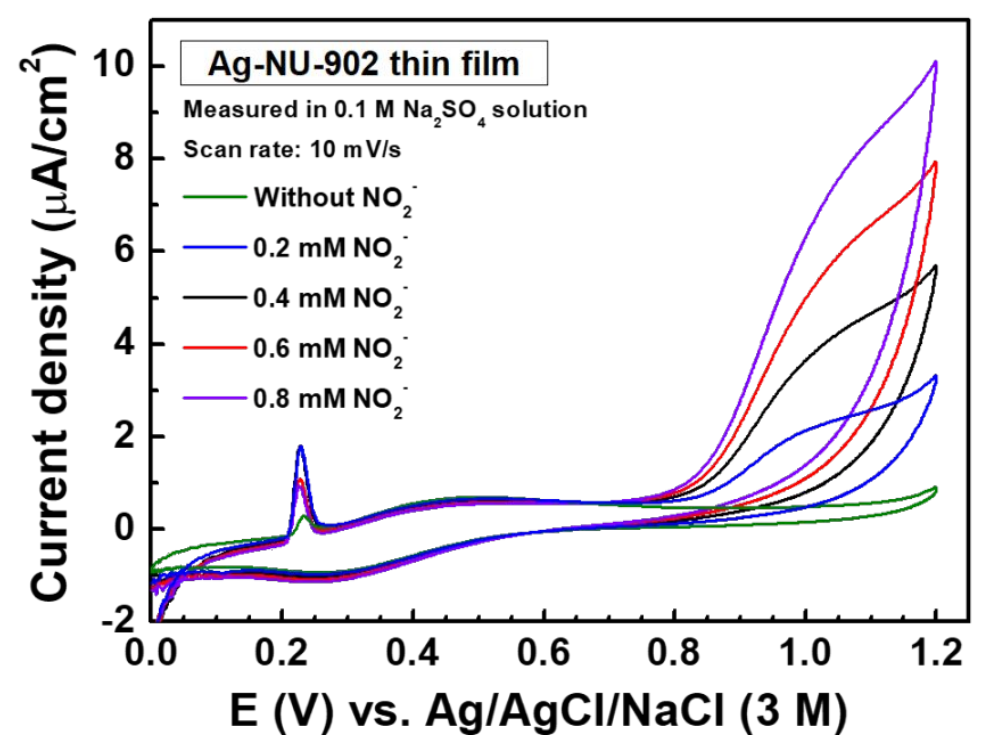

Figure S12. $\mathrm{CV}$ curves of the Ag-NU-902 thin film measured in the $0.1 \mathrm{M} \mathrm{Na}_{2} \mathrm{SO}_{4}$ aqueous solution containing various concentrations of nitrite. Scan rate: $10 \mathrm{mV} / \mathrm{s}$. 


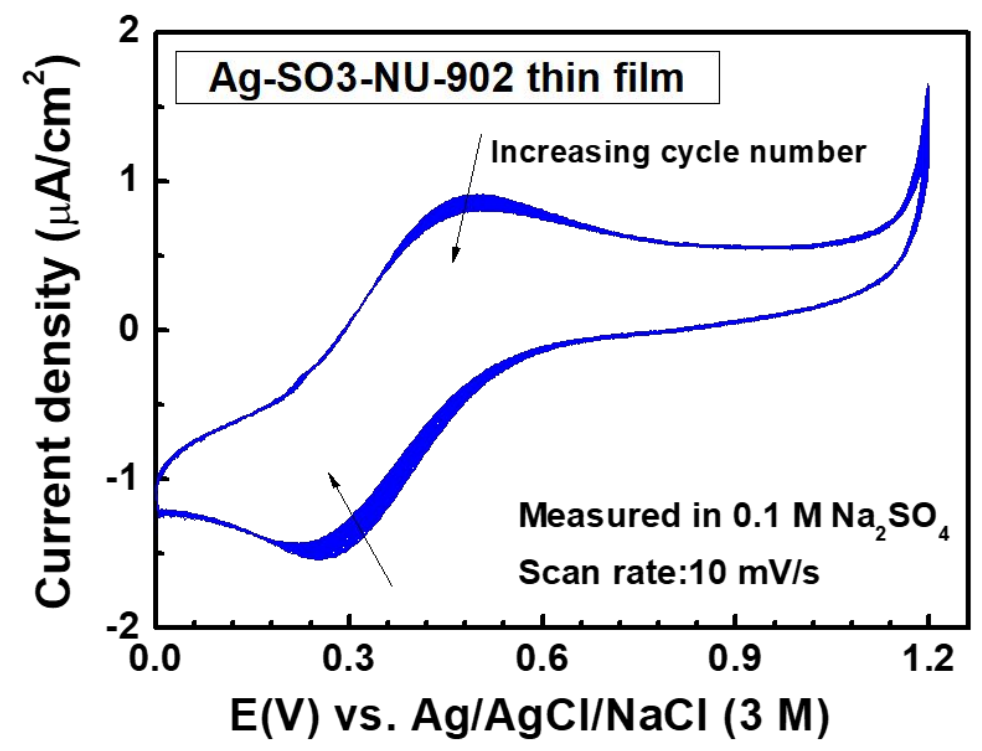

Figure S13. 100-cycle successive CV curves of the Ag-SO3-NU-902 thin film measured in the $0.1 \mathrm{M} \mathrm{Na}_{2} \mathrm{SO}_{4}$ aqueous solution at a scan rate of $10 \mathrm{mV} / \mathrm{s}$.

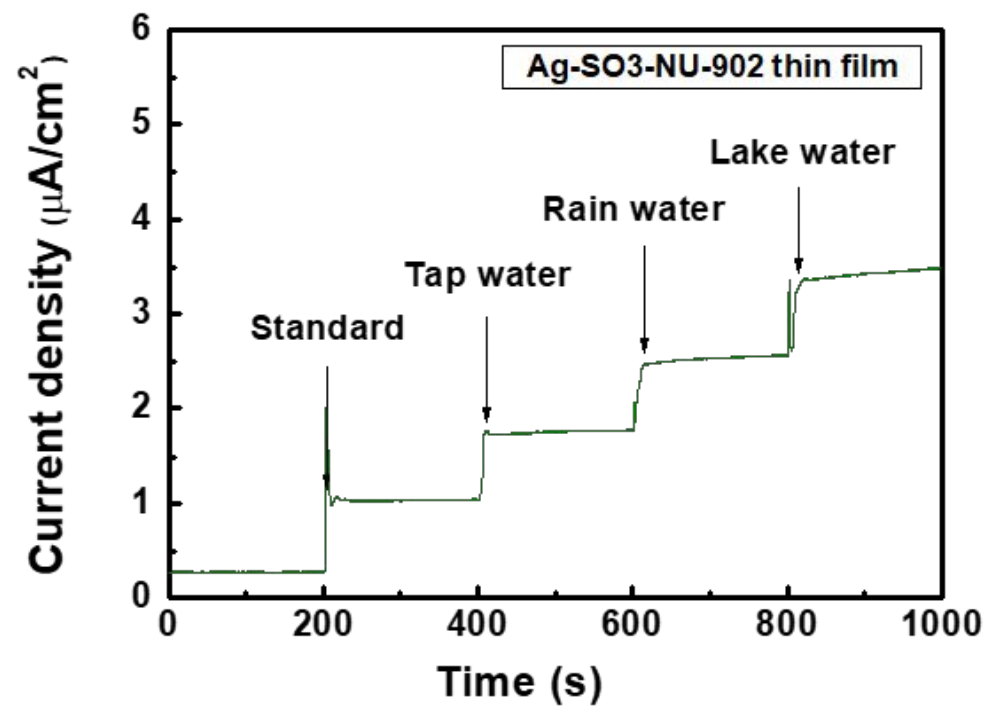

Figure S14. Representative amperometric curve of the Ag-SO3-NU-902 thin film recorded during successive additions of different real samples containing nitrite in the $0.1 \mathrm{M} \mathrm{Na}_{2} \mathrm{SO}_{4}$ aqueous solution. The increment in nitrite concentration in the bulk solution after each addition is $0.2 \mathrm{mM}$. 
Table S1. Partial list of the reported MOF-based electrochemical nitrite sensors compared to the Ag-SO3-NU-902 sensor.

\begin{tabular}{|c|c|c|c|}
\hline \multirow[b]{2}{*}{ Active material } & \multicolumn{2}{|c|}{ Performance } & \multirow[b]{2}{*}{ Reference } \\
\hline & $\begin{array}{l}\text { LOD } \\
(\mu \mathrm{M})\end{array}$ & $\begin{array}{l}\text { Linear range } \\
\text { (up to, } \mathrm{mM} \text { ) }\end{array}$ & \\
\hline Mb/Ag@Zn-TSA/IL-CP ${ }^{a}$ & 0.5 & 133 & {$[4]$} \\
\hline $\mathrm{Cu}-\mathrm{MOF} / \mathrm{rGO}$ hybrid $^{b}$ & 0.033 & 40 & [5] \\
\hline GQD-PCN-222 ${ }^{c}$ & 6.4 & 18 & [6] \\
\hline $\mathrm{Cu}-\mathrm{MOF} / \mathrm{Au} / \mathrm{GCE}^{d}$ & 0.082 & 10 & [7] \\
\hline GC/Au-MOF-5 & 15.3 & 6 & [8] \\
\hline IL/Cyt c/CoTCPP-CPE ${ }^{e}$ & 1.1 & 2.8 & [9] \\
\hline MOF-GNRs-50 ${ }^{f}$ & 0.75 & 2.5 & {$[10]$} \\
\hline Ag-SO3-NU-902 & 9.1 & 2 & This work \\
\hline 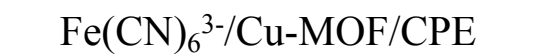 & 0.04 & 1.1 & [11] \\
\hline Cu-CoTCPP /MWCNTs/GCE $g$ & 0.17 & 1.1 & [12] \\
\hline M1.1 $h$ & 0.72 & 0.8 & [13] \\
\hline MOF-525 & 2.1 & 0.8 & [14] \\
\hline $\mathrm{Au} / \mathrm{Cu}-\mathrm{MOF} / \mathrm{CPE}{ }^{i}$ & 0.03 & 0.7 & [15] \\
\hline
\end{tabular}

a $\mathrm{Zn}-\mathrm{TSA}=$ Zinc thiosalicylate; IL = Ionic liquid

${ }^{b} \mathrm{rGO}=$ Reduced graphene oxide

${ }^{c} \mathrm{GQD}=$ Graphene quantum dots

${ }^{d} \mathrm{GCE}=$ Glassy carbon electrode

${ }^{e}$ Cyt $\mathrm{c}=$ Cytochrome $\mathrm{c}$

${ }^{f}$ GNRs $=$ Graphene nanoribbons

${ }^{g}$ MWCNTs $=$ Multi-walled carbon nanotube

${ }^{h} \mathrm{M} 1.1=$ Inkjet printed MOF-525 synthesized by adding $1.1 \mathrm{~g}$ of benzoic acid

${ }^{i} \mathrm{CPE}=$ Carbon paste electrode 
Table S2. Summarized results for the detection of nitrite in environmental water samples by using the Ag-SO3-NU-902-based sensor.

\begin{tabular}{ccccc}
\hline & Added $(\mathrm{mM})$ & Found $(\mathrm{mM})^{\mathrm{a}}$ & ${\text { Recovery }(\%)^{\mathrm{a}}}$ & R.S.D. $(\%)^{\mathrm{b}}$ \\
\hline Tap water & 0.2 & 0.174 & 87.0 & 16 \\
Rainwater & 0.2 & 0.202 & 101.0 & 23 \\
Lake water & 0.2 & 0.193 & 96.5 & 24 \\
\hline
\end{tabular}

a Averaged from three separated experiments

${ }^{\mathrm{b}}$ Relative standard deviation 


\section{References:}

1. Li, L.; Shen, S.; Lin, R.; Bai, Y.; Liu, H., Rapid and Specific Luminescence Sensing of $\mathrm{Cu}(\mathrm{Ii})$ Ions with a Porphyrinic Metal-Organic Framework. Chem. Commun. 2017, 53, 99869989.

2. Afzal, S.; Daoud, W. A.; Langford, S. J., Self-Cleaning Cotton by Porphyrin-Sensitized Visible-Light Photocatalysis. J. Mater. Chem. 2012, 22, 4083-4088.

3. Zheng, W.; Shan, N.; Yu, L.; Wang, X., Uv-Visible, Fluorescence and Epr Properties of Porphyrins and Metalloporphyrins. Dyes and Pigments 2008, 77, 153-157.

4. Jia, W.; Guo, M.; Zheng, Z.; Yu, T.; Rodriguez, E. G.; Wang, Y.; Lei, Y., Electrocatalytic Oxidation and Reduction of $\mathrm{H} 2 \mathrm{o} 2$ on Vertically Aligned Co3o4 Nanowalls Electrode: Toward H2o2 Detection. J. Electroanal. Chem. 2009, 625, 27-32.

5. Umesh, N. M.; rani, K. K.; Devasenathipathy, R.; Sriram, B.; Liu, Y.-X.; Wang, S.-F., Preparation of Co-Mof Derived $\mathrm{Co}(\mathrm{Oh}) 2 /$ Multiwalled Carbon Nanotubes as an Efficient Bifunctional Electro Catalyst for Hydrazine and Hydrogen Peroxide Detections. J. Taiwan Inst. Chem. Eng. 2018, 93, 79-86.

6. Wang, M.; Jiang, X.; Liu, J.; Guo, H.; Liu, C., Highly Sensitive H2o2 Sensor Based on Co3o4 Hollow Sphere Prepared Via a Template-Free Method. Electrochim. Acta 2015, 182, 613-620.

7. Wu, Z.; Sun, L.-P.; Zhou, Z.; Li, Q.; Huo, L.-H.; Zhao, H., Efficient Nonenzymatic H2o2 Biosensor Based on Zif-67 Mof Derived Co Nanoparticles Embedded N-Doped Mesoporous Carbon Composites. Sens. Actuators B Chem. 2018, 276, 142-149.

8. Liu, M.; He, S.; Chen, W., Co3o4 Nanowires Supported on 3d N-Doped Carbon Foam as an Electrochemical Sensing Platform for Efficient H2o2 Detection. Nanoscale 2014, 6, 11769-11776.

9. Xia, S.; Yu, M.; Hu, J.; Feng, J.; Chen, J.; Shi, M.; Weng, X., A Model of InterfaceRelated Enhancement Based on the Contrast between Co3o4 Sphere and Cube for Electrochemical Detection of Hydrogen Peroxide. Electrochem. Commun. 2014, 40, 67-70. 10. Lee, K. K.; Loh, P. Y.; Sow, C. H.; Chin, W. S., Coooh Nanosheet Electrodes: Simple 
Fabrication for Sensitive Electrochemical Sensing of Hydrogen Peroxide and Hydrazine. Biosens. Bioelectron. 2013, 39, 255-260.

11. Balamurugan, J.; Thanh, T. D.; Karthikeyan, G.; Kim, N. H.; Lee, J. H., A Novel Hierarchical 3d N-Co-Cnt@Ng Nanocomposite Electrode for Non-Enzymatic Glucose and Hydrogen Peroxide Sensing Applications. Biosens. Bioelectron. 2017, 89, 970-977.

12. Shi, L.-M.; Pan, J.-X.; Zhou, B.; Jiang, X., A New Bifunctional Electrochemical Sensor for Hydrogen Peroxide and Nitrite Based on a Bimetallic Metalloporphyrinic Framework. $J$. Mater. Chem. B 2015, 3, 9340-9348.

13. Su, C.-H.; Kung, C.-W.; Chang, T.-H.; Lu, H.-C.; Ho, K.-C.; Liao, Y.-C., Inkjet-Printed Porphyrinic Metal-Organic Framework Thin Films for Electrocatalysis. J. Mater. Chem. A 2016, 4, 11094-11102.

14. Kung, C.-W.; Chang, T.-H.; Chou, L.-Y.; Hupp, J. T.; Farha, O. K.; Ho, K.-C., Porphyrin-Based Metal-Organic Framework Thin Films for Electrochemical Nitrite Detection. Electrochem. Commun. 2015, 58, 51-56.

15. Yuan, B.; Zhang, J.; Zhang, R.; Shi, H.; Wang, N.; Li, J.; Ma, F.; Zhang, D., Cu-Based Metal-Organic Framework as a Novel Sensing Platform for the Enhanced Electro-Oxidation of Nitrite. Sens. Actuators B Chem. 2016, 222, 632-637. 